\title{
INFINITE PRIMES AND UNIQUE FACTORIZATION IN A PRINCIPAL RIGHT IDEAL DOMAIN $\left({ }^{1}\right)$
}

\author{
BY \\ RAYMOND A. BEAUREGARD $\left({ }^{2}\right)$
}

1. Introduction. Throughout this paper all rings are assumed to be integral domains with unity. If $R$ is an integral domain $R^{*}$ will denote the set of nonzero elements of $R$. An integral domain in which the sum and intersection of any two principal right ideals is principal whenever the intersection is nonzero is called a weak Bézout domain. In particular, if we do not require that the intersection be nonzero then $R$ is called a right Bézout domain (see [2]). If $R$ is a weak Bézout domain and $a \in R^{*}$ then the set $[a R, R]$ of all principal right ideals of $R$ that contain $a R$ is a sublattice of the lattice of all right ideals of $R$. In this case $\operatorname{dim} a$ is defined to be the dimension of the lattice $[a R, R]$, i.e., $\operatorname{dim} a$ is the length of the longest chain in $[a R, R]$. We let $R^{\prime}$ denote the set of finite dimensional elements of $R$. If each right ideal of an integral domain $R$ is a principal right ideal then $R$ is called a PRI (principal right ideal) domain. Thus each PRI domain is a weak Bézout domain.

The known types of unique factorization that occur in a PRI domain $R$ deal with the members of $R^{\prime}$. For example, it is well known [4, p. 34] that each nonunit $z$ of $R^{\prime}$ can be factored into primes: $z=p_{1} \cdots p_{n}$, and if $z=q_{1} \cdots q_{m}$ is another such factorization, then $n=m$ and there is a permutation $\Pi$ on $\{1,2, \ldots, n\}$ such that $p_{i}$ and $q_{\Pi(i)}$ are similar, $i=1,2, \ldots, n$. There is another type of unique factorization that occurs in $R^{\prime}$ which is described by R. E. Johnson in [6]. An element $a \in R^{\prime}$ is called simple if $[a R, R]$ has the property that $[a R, R]=[a R, B] \cup[B, R]$ implies $B=a R$ or $B=R$. Johnson proves that each element $z$ in $R^{\prime}$ can be factored into simple elements: $z=a_{1} \cdots a_{n}$, and no subproduct $a_{i} \cdots a_{j}, i<j$, of $z$ is simple. Any other factorization of $z$ into simple elements of this type must have the form $z=\left(a_{1} u_{1}\right)\left(u_{1}^{-1} a_{2} u_{2}\right) \cdots\left(u_{n-1}^{-1} a_{n}\right)$ where $u_{1}, \ldots, u_{n-1}$ are units in $R$.

The type of unique factorization that we describe in the present paper concerns all of the nonzero elements of a PRI domain $R$. In $\$ 2$ we develop some general results (in particular Theorem 2 ) for weak Bézout domains that satisfy the ascending chain condition for principal right ideals. In $\$ 3$ we define inf ${ }^{(\alpha)}$ primes for each nonlimit ordinal $\alpha . \operatorname{Inf}^{(0)}$ primes are the usual primes, and inf ${ }^{(\alpha)}$ primes have infinite dimension if $\alpha \neq 0$. The unique factorization theorem (Theorem 3) that follows

Received by the editors June 28, 1968 and, in revised form, November 12, 1968.

(1) This work is part of the author's Ph.D. dissertation which was done at the University of New Hampshire, Durham, New Hampshire.

$\left({ }^{2}\right)$ The author's present address is at the University of Rhode Island, Kingston, Rhode Island. 
states that each nonzero element $a$ of a PRI domain $R$ can be factored as $a=z_{\alpha_{1}}$ $\cdots z_{\alpha_{n}}$ where $z_{\alpha_{i}}$ is a uniquely determined product of $\inf ^{\left(\alpha_{i}\right)}$ primes. The factorization of $a$ is unique in the sense that if $a=y_{\beta_{1}} \cdots y_{\beta_{m}}$ is another such factorization of $a$ then $n=m, \alpha_{i}=\beta_{i}(i=1,2, \ldots, n)$, and there are units $u_{1}, \ldots, u_{n-1}$ in $R$ such that $z_{\alpha_{1}}=y_{\alpha_{1}} u_{1}, z_{\alpha_{n}}=u_{n-1}^{-1} y_{\alpha_{n}}$, and $z_{\alpha_{i}}=u_{i-1}^{-1} y_{\alpha_{i}} u_{i}(i \neq 1, n)$.

The important applications of Theorem 3 obviously occur in PRI domains $R$ such that $R^{*} \neq R^{\prime}$. Until recently few examples of such were available. Such examples however do occur, for instance, in P. M. Cohn [3]. In [5] A. V. Jategaonkar describes the method of skew polynomial extensions. Using these methods it is possible to construct PRI domains with inf ${ }^{(\alpha)}$ primes, given an arbitrary ordinal $\alpha$. We include this discussion in $\$ 4$.

2. Right quotient monoids. Suppose $R$ is an integral domain and $\varnothing \neq S \subset R^{*}$. We call $S$ a right quotient monoid in $R$ if $S$ satisfies the following right quotient conditons:

(1) $a b \in S$ iff $a$ and $b \in S$, where $a, b \in R$.

(2) $a \in R, b \in S$ implies there exists $\bar{a} \in R, \bar{b} \in S$ such that $a \bar{b}=b \bar{a}$.

In this case $S$ contains the group of units of $R$. For, $a \in S$ for some element $a$ of $R$. From $1 a \in S$ we obtain $1 \in S$ by condition (1). Also, if $u$ is a unit in $R$ then $u u^{-1}=$ $1 \in S$ and therefore $u \in S$.

If $S$ is a right quotient monoid in $R$ then the set $K=R S^{-1}=\left\{r s^{-1} \mid r \in R, s \in S\right\}$ can be made into a ring in the usual way (see Bourbaki $\left[1\right.$, p. 162])( $\left.{ }^{3}\right)$. It is easy to prove that $K$ is an integral domain with the property that $s \in R$ is a unit in $K$ iff $s \in S$. Further, if $A$ is a right ideal of $R$ then $A S^{-1}=\left\{a s^{-1} \mid a \in A, s \in S\right\}$ is a right ideal of $K$, and if $R$ is a PRI domain then so is $K$. The easy proofs of these facts are omitted.

LEMMA 1. Let $R$ be an integral domain and let $S$ be a right quotient monoid in $R$. Let $A, B$ be right ideals of $R$. Then

(1) $(A \cap B) S^{-1}=A S^{-1} \cap B S^{-1}$.

(2) $(A+B) S^{-1}=A S^{-1}+B S^{-1}$.

Proof. Clearly $(A \cap B) S^{-1} \subset A S^{-1} \cap B S^{-1}$. Now suppose $a s_{1}^{-1}=b s_{2}^{-1} \in A S^{-1}$ $\cap B S^{-1}\left(s_{1}, s_{2} \in S\right.$ and $\left.a, b \in R\right)$. Because $S$ is a right quotient monoid we can choose $\bar{s}_{1}, \bar{s}_{2} \in S$ such that $s_{1} \bar{s}_{2}=s_{2} \bar{s}_{1}$. It follows that $a \bar{s}_{2}=b \bar{s}_{1} \in A \cap B$. Also, $a s_{1}^{-1}=a \bar{s}_{2}\left(s_{2} \bar{s}_{1}\right)^{-1} \in(A \cap B) S^{-1}$. This proves $A S^{-1} \cap B S^{-1} \subset(A \cap B) S^{-1}$ and (1) is established.

Now it is obvious that $(A+B) S^{-1} \subset A S^{-1}+B S^{-1}$. To show the reverse inclusion let $a s_{1}^{-1}+b s_{\mathrm{e}}^{-1} \in A S^{-1}+B S^{-1}\left(s_{1}, s_{2} \in S\right.$ and $\left.a, b \in R\right)$. Choose $\bar{s}_{1}, \bar{s}_{2} \in S$ such that $s_{1} \bar{s}_{2}=s_{2} \bar{s}_{1}$. Then $a s_{1}^{-1}+b s_{2}^{-1}=\left(a \bar{s}_{2}+b \bar{s}_{1}\right)\left(s_{1} \bar{s}_{2}\right)^{-1}$. Hence $a s_{1}^{-1}+b s_{2}^{-1} \in(A+B) S^{-1}$. This proves (2). Q.E.D.

$\left({ }^{3}\right)$ For this purpose condition (1) in the definition of right quotient monoid is usually replaced by the weaker condition that $S$ be multiplicatively closed. 
CoRollary. If $R$ is a weak Bézout or a right Bézout domain, then so is $K=R S^{-1}$

We recall from [2] that if $a, \bar{a} \in R$, then $a$ and $\bar{a}$ are similar $\left(a \sim \bar{a}\right.$ or $\left.a \sim_{R} \bar{a}\right)$ if $R / a R \cong R / \bar{a} R$ as right $R$-modules, and this is true iff there exists $b \in R$ such that $a R+b R=R$ and $a R \cap b R=b \bar{a} R$. It is also shown in [2] that the definition of similarity is left-right symmetric.

LEMMA 2. Let $S$ be a right quotient monoid in an integral domain $R$ and let $K=R S^{-1}$. If $a, \bar{a} \in R$ and $a \sim_{R} \bar{a}$ then $a \sim_{K} \bar{a}$. Further, if $a \sim_{R} \bar{a}$ and $a \in S$ then $\bar{a} \in S$.

Proof. Let $a, \bar{a} \in R$ with $a \sim_{R} \bar{a}$. Then $a R+b R=R$ and $a R \cap b R=b \bar{a} R$ for some $b \in R$. Therefore $a K+b K=K$ and $a K \cap b K=b \bar{a} K$ by Lemma 1. Hence $a \sim_{K} \bar{a}$. If in addition $a \in S$ then $a$ is a unit in $K$. Since $a \sim_{K} \bar{a}$ it follows that $\bar{a}$ is a unit in $K$. Hence $\bar{a} \in S$. Q.E.D.

Lemma 3. Suppose $R$ is a right Bézout domain and $\varnothing \neq S \subset R^{*}$ such that ab $\in S$ iff $a$ and $b \in S$. Then $S$ is a right quotient monoid in $R$ iff elements similar to members of $S$ belong to $S$, that is, iff $a \in S, \bar{a} \in R$, and $a \sim \bar{a}$ implies $\bar{a} \in S$.

Proof. Assume the hypotheses. If $S$ is a right quotient monoid in $R$ then elements similar to members of $S$ belong to $S$ by Lemma 2 . Conversely assume that $S$ has the property that $a \in S, \bar{a} \in R$, and $a \sim \bar{a}$ implies $\bar{a} \in S$. To show that $S$ is a right quotient monoid in $R$ we need only establish condition (2) of the definition. Accordingly let $a \in S$ and $b \in R$. Because $R$ is a right Bézout domain we can choose $d, m \in R$ such that $a R+b R=d R$ and $a R \cap b R=m R$. Then $a=d a^{\prime}, b=d b^{\prime}$, and $m=a \bar{b}=b \bar{a}$ for some $a^{\prime}, b^{\prime}, \bar{a}, \bar{b} \in R$. It follows that $a^{\prime} R+b^{\prime} R=R$ and $a^{\prime} R \cap b^{\prime} R$ $=b^{\prime} \bar{a} R$. Therefore $a^{\prime} \sim \bar{a}$. Now $a \in S$ implies $a^{\prime} \in S$. Hence $\bar{a} \in S$ by our assumption. This shows that $S$ is a right quotient monoid in $R$. Q.E.D.

COROllaRY. If $R$ is a right Bézout domain then $R^{*}$ is a right quotient monoid in $R$.

THEOREM 1. Let $R$ be a weak Bézout domain satisfying the ascending chain condition for principal right ideals, and let $S$ be a right quotient monoid in $R$. Each $z \in R^{*}$ can be written as $z=x s$ where $s \in S$ and $x$ has no nonunit right factor in $S$. The factorization is unique in the sense that if $z=x_{1} s_{1}=x_{2} s_{2}$ are two such factorizations of $z$ then there is a unit $u \in R$ such that $x_{1}=x_{2} u\left(\right.$ and $\left.u s_{1}=s_{2}\right)$.

Proof. Let $z \in R^{*}$. Let $C_{S}$ be the collection of submodules $R / s R$ of $R / z R$ such that $s \in S$. Note that $z R / z R \cong R / R$ is a member of $C_{S}$. We claim that $C_{S}$ is closed under sums. For if $a R / z R, b R / z R \in C_{S}$, then $a R / z R \cong R / x R, b R / z R \cong R / y R$ where $z=a x=b y$ and $x, y \in S$. Since $R$ is a weak Bézout domain, $a R+b R=d R, a R \cap b R$ $=m R$ for some $d, m \in R$. Choose $a^{\prime}, b^{\prime}, \bar{a}, \bar{b} \in R$ with $a=d a^{\prime}, b=d b^{\prime}, m=a \bar{b}=b \bar{a}$. Then $a^{\prime} R+b^{\prime} R=R, a^{\prime} R \cap b^{\prime} R=b^{\prime} \bar{a} R$. Hence $a^{\prime} \sim \bar{a}$. Now $a x=b y \in m R$ implies 
$b y=m r$ for some $r \in R$. Hence $y=\bar{a} r$. This shows that $\bar{a} \in S$ because $y \in S$. Consequently $a^{\prime} \in S$. Then $a R / z R+b R / z R=d R / z R=d R / d a^{\prime} x R \cong R / a^{\prime} x R$ and $a^{\prime} x \in S$. We conclude $a R / z R+b R / z R \in C_{S}$.

To prove the theorem observe that $C_{S}$ has the ascending chain condition by hypothesis. Thus we may select a (not necessarily proper) maximal member $x R / z R$ of $C_{S}$. Thus $x R / z R \cong R / s R$ where $z=x s$ and $s \in S$. Since $x R / z R$ is maximal $x$ has no nonunit right factor in $S$. Also, $x R / z R$ is the unique maximal member of $C_{S}$ because $C_{S}$ is closed under sums. Therefore the factorization is unique. Q.E.D.

Let $R$ be an integral domain and let $I=\left\{\alpha \mid 0 \leqq \alpha \leqq \alpha_{0}\right\}$ be an initial segment of ordinals. A collection $\left\{S_{\alpha} \mid \alpha \in I\right\}$ of right quotient monoids in $R$ is called a right quotient chain in $R$ if the following conditions hold:

(1) $S_{\alpha} \subsetneq S_{\alpha+1}$ for each $\alpha \in I, \alpha \neq \alpha_{0}$.

(2) $S_{\alpha}=\bigcup_{\beta<\alpha} S_{\beta}$ if $\alpha$ is a limit ordinal.

For convenience we let $S_{-1}$ denote the group of units of $R$. Then $S_{-1}$ is contained in each $S_{\alpha}$. Let $K_{\alpha}=R\left(S_{\alpha}\right)^{-1}$ if $\alpha=-1$ or if $\alpha \in I$. Then because of condition (1) $K_{\alpha-1} \subset K_{\alpha}$ for each $\alpha \in I$.

THEOREM 2. Let $R$ be a weak Bézout domain satisfying the ascending chain condition for principal right ideals. Let $I=\left\{\alpha \mid 0 \leqq \alpha \leqq \alpha_{0}\right\}$ be an initial segment of ordinals and let $\left\{S_{\alpha} \mid \alpha \in I\right\}$ be a right quotient chain in $R$. Each $z \in R^{*}$ can be factored as $z=r a_{\alpha_{1}} \cdots a_{\alpha_{n}}$ where $\alpha_{i}$ are nonlimit ordinals such that $\alpha_{0} \geqq \alpha_{1}>\cdots>\alpha_{n}, a_{\alpha_{i}} \in S_{\alpha_{i}}$, $a_{\alpha_{i}}$ has no nonunit right factor in $S_{\alpha_{i}-1}, r \in R$ and $r$ has no nonunit right factor in $S_{\alpha_{0}}$. The factorization is unique in the sense that if $z=s b_{\beta_{1}} \cdots b_{\beta_{m}}$ is another such factorization of $z$ then $n=m, \alpha_{i}=\beta_{i}(i=1,2, \ldots, n)$, and there are units $u_{0}, u_{1}, \ldots$, $u_{n-1}$ in $R$ such that $r=s u_{0}, a_{\alpha_{n}}=u_{n-1}^{-1} b_{\alpha_{n}}$, and $a_{\alpha_{i}}=u_{i-1}^{-1} b_{\alpha_{i}} u_{i}(i \neq 0, n)$.

Proof. To prove existence of the factorization let $z \in R^{*}$. If $z$ has no nonunit right factor in $S_{\alpha_{0}}$ then we are finished. Otherwise by Theorem $1 z=r s_{0}$ for some nonunit $s_{0} \in S_{\alpha_{0}}$ and $r$ has no nonunit right factor in $S_{\alpha_{0}}$. Let $\alpha_{1}$ be the least ordinal such that $s_{0} \in S_{\alpha_{1}}$. Clearly $\alpha_{1}$ is not a limit ordinal and $\alpha_{0} \geqq \alpha_{1}$. It follows by Theorem 1 that $s_{0}=a_{\alpha_{1}} s_{1}$ for some element $s_{1} \in S_{\alpha_{1}-1}$ and $a_{\alpha_{1}}$ has no nonunit right factor in $S_{\alpha_{1}-1}$. Clearly $a_{\alpha_{1}} \in S_{\alpha_{1}}$ because $s_{0} \in S_{\alpha_{1}}$. If $s_{1}$ is not a unit let $\alpha_{2}$ be the least ordinal such that $s_{1} \in S_{\alpha_{2}}$. Then $\alpha_{1}>\alpha_{2}$ and $\alpha_{2}$ is not a limit ordinal. Another application of Theorem 1 yields $s_{1}=a_{\alpha_{2}} s_{2}$ where $s_{2} \in S_{\alpha_{2}-1}$ and $a_{\alpha_{2}}$ has no nonunit right factor in $S_{\alpha_{2}-1}$. Clearly $a_{\alpha_{2}} \in S_{\alpha_{2}}$ because $s_{1} \in S_{\alpha_{2}}$. If $s_{2}$ is not a unit we may repeat the argument. Now this process cannot continue indefinitely since we would obtain an infinite sequence $\alpha_{1}>\alpha_{2}>\cdots$ contradicting the well ordering of the ordinals. Thus the procèss stops, say, with the integer $n$. That is, $a_{\alpha_{n}}$ has no nonunit right factor in $S_{\alpha_{n}-1}$ and $s_{n}$ is a unit. This establishes the existence of the factorization.

To prove uniqueness suppose $z=r a_{\alpha_{1}} \cdots a_{\alpha_{n}}=s b_{\beta_{1}} \cdots b_{\beta_{m}}$ are two factorizations of $z$ of the type stated in the theorem. Then Theorem 1 applies and yields $r=s u_{0}$ for some unit $u_{0} \in R$. Therefore $a_{\alpha_{1}} \cdots a_{\alpha_{n}}=u_{0}^{-1} b_{\beta_{1}} \cdots b_{\beta_{m}}$. Evidently $\alpha_{1}=\beta_{1}$. Again Theorem 1 applies and yields $a_{\alpha_{1}}=u_{0}^{-1} b_{\beta_{1}} u_{1}$ for some unit $u_{1} \in R$. Cancelling this 
factor we obtain $a_{\alpha_{2}} \cdots a_{\alpha_{n}}=u_{1}^{-1} b_{\beta_{2}} \cdots b_{\beta_{m}}$. Uniqueness now follows by induction. Q.E.D.

3. Unique factorization and infinite primes. In this section we shall construct a natural set $\left\{R^{(\alpha)} \mid \alpha \in I\right\}$ which is a right quotient chain in a right Bézout domain $R$. We shall then apply Theorem 2 to this right quotient chain. We begin by characterizing the peculiar factors that appear in Theorem 2 .

Let $I=\left\{\alpha \mid 0 \leqq \alpha \leqq \alpha_{0}\right\}$ be an initial segment of ordinals and let $\left\{S_{\alpha} \mid \alpha \in I\right\}$ be a right quotient chain in an integral domain $R$. If $\alpha$ is a nonlimit ordinal in $I$ then $x \in S_{\alpha}$ is called an $\alpha$-prime if $x R$ is maximal in $\left\{x R\left|x \in S_{\alpha}\right| S_{\alpha-1}\right\}$.

Lemma 4. Let $I=\left\{\alpha \mid 0 \leqq \alpha \leqq \alpha_{0}\right\}$ be an initial segment of ordinals and let $\left\{S_{\alpha} \mid \alpha \in I\right\}$ be a right quotient chain in a PRI domain $R$. If $\alpha$ is a nonlimit ordinal in $I$ and $x$ is an $\alpha$-prime then $x$ is prime in $K_{\alpha-1}$.

Proof. Assume the hypotheses and let $x$ be an $\alpha$-prime. Suppose $x K_{\alpha-1} \subsetneq y K_{\alpha-1}$ $\subset K_{\alpha-1}$. Then $x R \subset x K_{\alpha-1} \cap R \subsetneq y K_{\alpha-1} \cap R \subset R$. Let $\bar{y} \in R$ be such that $\bar{y} R$ $=y K_{\alpha-1} \cap R$. Then $x R \subsetneq \bar{y} R$. The definition of $\alpha$-prime implies that $\bar{y} \in S_{\alpha-1}$ and therefore $\bar{y}$ is a unit in $K_{\alpha-1}$ and $\bar{y} K_{\alpha-1}=K_{\alpha-1}$. Now $\bar{y} K_{\alpha-1}=y K_{\alpha-1}$ and so $y K_{\alpha-1}=K_{\alpha-1}$. This shows that $x$ is prime in $K_{\alpha-1}$. Q.E.D.

Lemma 5. Let $R$ be a weak Bézout domain, let I be an initial segment of ordinals, and let $\left\{S_{\alpha} \mid \alpha \in I\right\}$ be a right quotient chain in $R$. If $x_{1}, \ldots, x_{k}$ are $\alpha$-primes, then $x_{1} \cdots x_{k}$ has no nonunit right factor that belongs to $S_{\alpha-1}$.

Proof. The proof is by induction on $k$. The lemma is true if $k=1$ by the definition of $\alpha$-prime. Assume $k$ is an integer greater than 1 and the lemma holds for positive integers less than $k$. Suppose $x_{1} \cdots x_{k}=a b$ with $b \in S_{\alpha-1}, a \in R$ and $x_{i}$ are $\alpha$-primes. We shall show that $b$ must be a unit. If $a R \subset x_{1} R$ then $a=x_{1} s, s \in R$. Hence $x_{2} \cdots x_{k}$ $=s b$. It follows by induction that $b$ must be a unit. Suppose on the other hand that $a R \notin x_{1} R$. Then since $x_{1} R \cap a R \neq 0$ and $R$ is a weak Bézout domain it follows that $x_{1} R+a R=d R$ and $x_{1} R \cap a R=m R$ for some $d, m \in R$. Choose $x^{\prime}, a^{\prime}, \bar{a}$, $\bar{x}_{1} \in R$ such that $x_{1}=d x^{\prime}, a=d a^{\prime}$, and $m=x_{1} \bar{a}=a \bar{x}_{1}$. Then $x^{\prime} R+a^{\prime} R=R$ and $x^{\prime} R$ $\cap a^{\prime} R=a^{\prime} \bar{x}_{1} R$. Consequently $x^{\prime} \sim \bar{x}_{1}$. Now $x_{1} \cdots x_{k}=x_{1} \bar{a} z$ for some $z \in R$. Therefore $a b=x_{1} \cdots x_{k}=x_{1} \bar{a} z$ and so $b=\bar{x}_{1} z$. Hence $z \in S_{\alpha-1}$ since $b \in S_{\alpha-1}$. It follows from $x_{2} \cdots x_{k}=\bar{a} z$ and by induction that $z$ is a unit. Consequently $b$ is a right associate of $\bar{x}_{1}$. Therefore $x^{\prime} \sim \bar{x}_{1}$ yields $x^{\prime} \sim b$ and hence $x^{\prime} \in S_{\alpha-1}$ by Lemma 2 . Now $d \in S_{\alpha-1}$ because $x_{1} R \subsetneq d R$ and $x_{1}$ is an $\alpha$-prime. Therefore $x_{1}=d x^{\prime} \in S_{\alpha-1}$. However this contradicts the fact that $x_{1}$ is an $\alpha$-prime. Q.E.D.

Whenever $R$ is a weak Bézout domain satisfying the ascending chain condition for principal right ideals the converse to Lemma 5 holds as follows.

LEMMA 6. Let $R$ be a weak Bézout domain satisfying the ascending chain condition for principal right ideals. Let I be an initial segment of ordinals and let $\left\{S_{\alpha} \mid \alpha \in I\right\}$ be a right quotient chain in $R$. Let $\alpha$ be a nonlimit ordinal in I, and let $a \in S_{\alpha} \cap\left(K_{\alpha-1}\right)^{\prime}$. If a has no nonunit right factor that belongs to $S_{\alpha-1}$ then $a$ is a product of $\alpha$-primes. 
Proof. Assume the hypotheses. Since $a \in S_{\alpha} \mid S_{\alpha-1}$ we may choose $x_{1}$ (by the ascending chain condition for principal right ideals) such that $x_{1} R$ is maximal in $\left\{x R \mid a R \subset x R\right.$ and $\left.x \in S_{\alpha} \mid S_{\alpha-1}\right\}$. Then $x_{1}$ is an $\alpha$-prime and $a=x_{1} s_{1}$ for some $s_{1} \in R$. Clearly $s_{1} \in S_{\alpha}$ and if $s_{1}$ is not a unit then $s_{1} \in S_{\alpha} \mid S_{\alpha-1}$ because of the assumption on $a$. We repeat the argument and obtain $s_{1}=x_{2} s_{2}$ where $x_{2}$ is an $\alpha$-prime and $s_{2} \in S_{\alpha}$. If this process does not terminate we obtain, for each positive integer $i, s_{i}=x_{i+1} s_{i+1}$ where $x_{i+1}$ is an $\alpha$-prime and hence a nonunit in $K_{\alpha-1}$. Let $r_{i}=x_{1} \cdots x_{i}$. Then $a=r_{i} s_{i}$ and $r_{i+1}=r_{i} x_{i+1}$ for each positive integer $i$. Therefore $a K_{\alpha-1} \subsetneq \cdots \subsetneq r_{i+1} K_{\alpha-1} \subsetneq r_{i} K_{\alpha-1} \subsetneq \cdots \subsetneq r_{1} K_{\alpha-1}$. This contradicts the hypothesis that $a \in\left(K_{\alpha-1}\right)^{\prime}$. Therefore the process terminates, say, with the integer $k$. Thus $a=x_{1} \cdots x_{k} s_{k}$ and $s_{k}$ is a unit in $R$. Q.E.D.

Each right Bézout domain contains a natural right quotient monoid as follows.

Lemma 7. Let $R$ be a right Bézout domain. Then $R^{\prime}$ is a right quotient monoid in $R$.

Proof. Clearly $\varnothing \neq R^{\prime} \subset R^{*}$. Suppose $a, b \in R^{*}$. Then $a R / a b R \cong R / b R$ and therefore $\operatorname{dim} a b=\operatorname{dim} a+\operatorname{dim} b$. Hence $a b \in R^{\prime}$ iff $a, b \in R^{\prime}$. Also if $a \in R^{\prime}$ and $\bar{a} \in R$ with $a \sim \bar{a}$ then $a R+b R=R$ and $a R \cap b R=b \bar{a} R$ for some $b \in R$. Therefore $[a R, R]$ $=[a R, a R+b R] \cong[a R \cap b R, b R]=[b \bar{a} R, b R] \cong[\bar{a} R, R]$ as lattices. Thus $\operatorname{dim} a$ $=\operatorname{dim} \bar{a}$ and so $\bar{a} \in R^{\prime}$. It follows by Lemma 3 that $R^{\prime}$ is a right quotient monoid in $R$. Q.E.D.

Let $R$ be a right Bézout domain. We construct, by transfinite induction, a natural chain $\left\{R^{(\alpha)} \mid \alpha\right.$ is an ordinal $\}$ of right quotient monoids in $R$ as follows.

Let $R^{(0)}=R^{\prime}$. Let $\alpha$ be an ordinal greater than zero and assume $R^{(\beta)}$ has been defined and is a right quotient monoid in $R$ whenever $\beta<\alpha$, and let $K_{\beta}=R\left(R^{(\beta)}\right)^{-1}$. Then $K_{\beta}$ is a right Bézout domain (Corollary to Lemma 1 ) and hence $K_{\beta}^{\prime}$ is a right quotient monoid in $K_{\beta}$ by Lemma 7 . We define $R^{(\alpha)}$ by

$$
\begin{aligned}
& R^{(\alpha)}=\bigcup_{\beta<\alpha} R^{(\beta)} \text { if } \alpha \text { is a limit ordinal, } \\
& R^{(\alpha)}=\left(K_{\alpha-1}\right)^{\prime} \cap R \text { if } \alpha \text { is not a limit ordinal. }
\end{aligned}
$$

To show that the induction is valid we must show that $R^{(\alpha)}$ is a right quotient monoid in $R$. If $\alpha$ is a limit ordinal the proof is obvious. Assume that $\alpha$ is not a limit ordinal. Clearly $\varnothing \neq R^{(\alpha)} \subset R^{*}$. Also $a b \in R^{(\alpha)}$ iff $a, b \in R^{(\alpha)}$ because $\left(K_{\alpha-1}\right)^{\prime}$ has this property. Now if $a \in R^{(\alpha)}, \bar{a} \in R$ and $a \sim_{R} \bar{a}$ then $a \sim_{K_{\alpha-1}} \bar{a}$ by Lemma 2 . It follows (as in the proof of Lemma 7) that $\operatorname{dim}_{K_{\alpha-1}} a=\operatorname{dim}_{K_{\alpha-1}} \bar{a}$. Hence $\bar{a} \in\left(K_{\alpha-1}\right)^{\prime}$ since $a \in\left(K_{\alpha-1}\right)^{\prime}$. It follows that $\bar{a} \in R^{(\alpha)}$. The hypotheses of Lemma 3 are satisfied and therefore $R^{(\alpha)}$ is a right quotient monoid in $R$. Q.E.D.

If $\alpha, \beta$ are ordinals such that $\alpha \leqq \beta$, then $R^{(\alpha)} \subset R^{(\beta)} \subset R$. Also $R^{(\alpha)}=R^{(\alpha+1)}$ for some ordinal $\alpha$. For if $R^{(\alpha)} \neq R^{(\alpha+1)}$ for each ordinal $\alpha$ then card $\left(R^{(\alpha)}\right) \geqq$ card $(\alpha)$ for each ordinal $\alpha$. Choosing $\beta$ such that card $(\beta)>\operatorname{card}(R)$ we obtain $\operatorname{card}(\beta)>\operatorname{card}(R)$ $\geqq$ card $\left(R^{(\beta)}\right)$, a contradiction. We let $\alpha_{0}$ denote the least ordinal such that $R^{\left(\alpha_{0}\right)}$ $=R^{\left(\alpha_{0}+1\right)}$, and we call $\left\{R^{(\alpha)} \mid 0 \leqq \alpha \leqq \alpha_{0}\right\}$ the right $D$-chain (Dimension chain) in $R$. In this situation $R^{(-1)}$ will denote the group of units of $R$. 
Evidently the right $D$-chain in a right Bézout domain $R$ is a right qotient chain in $R$. If $R$ is a PRI domain then the right $D$-chain has the following additional property.

LEMMA 8. Let $R$ be $a$ PRI domain and let $\left\{R^{(\alpha)} \mid 0 \leqq \alpha \leqq \alpha_{0}\right\}$ be the right $D$-chain in $R$. Then $R^{\left(\alpha_{0}\right)}=R^{*}$.

Proof. Suppose $R^{*} \neq R^{\left(\alpha_{0}\right)}$. Then by the maximum condition in the PRI domain $R$ we may choose $x$ such that $x R$ is maximal in $\left\{x R\left|x \in R^{*}\right| R^{\left(\alpha_{0}\right)}\right\}$. The proof of Lemma 4 can be used to show that $x$ is prime in $K_{\alpha_{0}}$. In particular $x \in\left(K_{\alpha_{0}}\right)^{\prime}$ $\cap R=R^{\left(\alpha_{0}+1\right)}$. This contradicts $R^{\left(\alpha_{0}+1\right)}=R^{\left(\alpha_{0}\right)}$. Therefore $R^{*}=R^{\left(\alpha_{0}\right)}$. Q.E.D.

Let $R$ be a PRI domain and let $\left\{R^{(\alpha)} \mid 0 \leqq \alpha \leqq \alpha_{0}\right\}$ be the right $D$-chain in $R$. We shall call the $\alpha$-primes in $R \inf ^{(\alpha)}$ primes. If $\alpha$ is a nonlimit ordinal such that $\alpha \leqq \alpha_{0}$ we let $Z^{(\alpha)}$ be the set of (finite) products of inf ${ }^{(\alpha)}$ primes.

If $R$ is a PRI domain and $\alpha$ is a nonlimit ordinal such that $\alpha \leqq \alpha_{0}$ then $x \in R$ is an $\inf ^{(\alpha)}$ prime iff $x R$ is maximal in $\left\{x R|x \in R| R^{(\alpha-1)}\right\}$. For if $x R$ is maximal in $\left\{x R|x \in R| R^{(\alpha-1)}\right\}$ then the proof of Lemma 4 can be used to show that $x$ is prime in $K_{\alpha-1}$. In particular $x \in\left(K_{\alpha-1}\right)^{\prime}$ and so $x \in R^{(\alpha)}$. Hence $x$ is an inf ${ }^{(\alpha)}$ prime. As a consequence we note that inf ${ }^{(\alpha)}$ primes exist for each nonlimit ordinal $\alpha \leqq \alpha_{0}$ by the maximum condition in $R$. In fact for each $z \notin R^{(\alpha-1)}, z R \subset x R$ for some $\inf ^{(\alpha)}$ prime $x$.

If $R$ is a PRI domain we can combine Lemmas 5 and 6 into the following.

LEMMA 9. Let $R$ be a PRI domain and let $\left\{R^{(\alpha)} \mid 0 \leqq \alpha \leqq \alpha_{0}\right\}$ be the right D-chain in $R$. Let $\alpha$ be a nonlimit ordinal such that $\alpha \leqq \alpha_{0}$, and let $z \in R^{(\alpha)}$. Then $z$ has no nonunit right factor in $R^{(\alpha-1)}$ iff $z \in Z^{(\alpha)}$, i.e., iff $z$ is a product of $\inf ^{(\alpha)}$ primes.

Using Lemmas 8 and 9 we can state Theorem 2 for the present case as follows.

TheOREM 3. Let $R$ be a PRI domain and let $\left\{R^{(\alpha)} \mid 0 \leqq \alpha \leqq \alpha_{0}\right\}$ be the right D-chain in $R$. Each $a \in R^{*}$ can be written as $a=z_{\alpha_{1}} \cdots z_{\alpha_{k}} u$ where $\alpha_{i}$ are nonlimit ordinals such that $\alpha_{0} \geqq \alpha_{1}>\cdots>\alpha_{k}$ and $z_{\alpha_{i}} \in Z^{\left(\alpha_{i}\right)}$ and $u$ is a unit in $R$. This factorization is unique in the sense that if $a=y_{\beta_{1}} \cdots y_{\beta_{h}} v$ is another such factorization of $a$ then $h=k$, $\alpha_{i}=\beta_{i}(i=1,2, \ldots, k)$, and there are units $u_{1}, \ldots, u_{k-1}$ in $R$ such that $z_{\alpha_{1}}=y_{\alpha_{1}} u_{1}$, $z_{\alpha_{k}}=u_{k-1}^{-1} y_{\alpha_{k}}$, and $z_{\alpha_{i}}=u_{i-1}^{-1} y_{\alpha_{i}} u_{i}(i \neq 1, k)$.

We note that the factors that appear in Theorem 3 are themselves uniquely determined as follows. Let $\alpha \in\left[0, \alpha_{0}\right]$ be a nonlimit ordinal and let $z \in Z^{(\alpha)}, z$ a nonunit. Then $z$ is a product of $\inf ^{(\alpha)}$ primes. Since an inf ${ }^{(\alpha)}$ prime is a prime in $K_{\alpha-1}$ (Lemma 4) the decomposition of $z$ is unique up to similarity in $K_{\alpha-1}$. That is, if $z=x_{1} \cdots x_{n}=y_{1} \cdots y_{m}$ where $x_{i}$ and $y_{i}$ are $\inf ^{(\alpha)}$ primes in $R$, then $n=m$ and there is a permutation $\Pi$ on $\{1,2, \ldots, n\}$ such that $x_{i} \sim_{K_{\alpha-1}} y_{\Pi(i)}(i=1,2, \ldots, n)$.

4. Example. If $L$ is a ring and $\sigma$ is a monomorphism from $L$ into $L$ we shall denote by $H=L[x, \sigma]$ the ring of skew polynomials in an indeterminate $x$ with coefficients in $L$ (written on he right of $x$ ). Addition in $H$ is the usual pointwise 
addition and multiplication is determined by the associative and distributive laws and by the commutation rule $a x=x a^{\sigma}(a \in L)$. It is easy to prove that $L$ is an integral domain iff $H$ is an integral domain (see Ore [7]). Further, it is shown in [5] that $H$ is a PRI domain iff $L$ is a PRI domain and $\sigma$ maps $L^{*}$ into the group of units of $L$.

We turn our attention to skew polynomial extensions which are defined by A. V. Jategaonkar in [5]. Let $\bar{\alpha}$ be an ordinal, let $I=[0, \bar{\alpha}]$, and let $L$ be an integral domain. Let $H$ be a right twisted polynomial extension of $L$ with $\left\{H_{(\alpha)} \mid \alpha \in I\right\}$ a chain of twisted subdomains from $L$ to $H$. Thus for each nonlimit ordinal $\alpha \in I$ there exists a monomorphism $\rho_{\alpha}: H_{(\alpha-1)} \rightarrow H_{(\alpha-1)}$ and an indeterminate $x_{\alpha}$ such that

$$
\begin{aligned}
H & =H_{(\tilde{\alpha})}, L=H_{(-1)}, \\
H_{(\alpha)} & =H_{(\alpha-1)}\left[x_{\alpha}, \rho_{\alpha}\right] \text { if } \alpha \in I \text { and } \alpha \text { is not a limit ordinal, } \\
H_{(\alpha)} & =\bigcup_{\beta<\alpha} H_{(\beta)} \text { if } \alpha \in I \text { and } \alpha \text { is a limit ordinal. }
\end{aligned}
$$

In addition we assume that $L$ is a skew field and $\rho_{\alpha}: H_{(\alpha-1)} \rightarrow L$ for each nonlimit ordinal $\alpha \in I$. Then $H$ is a PRI domain [5]. Now each member of $H$ is a polynomial in a finite number of indeterminates. Let $S$ be the set of polynomials of $H$ with nonzero constant terms in $L$. Then it can be shown [5] that $S$ is a right quotient monoid in $H$, and $R=H S^{-1}$ is a local PRI domain with unique maximal ideal $x_{0} R$ such that

$$
\begin{aligned}
& x_{\alpha+1} R=\bigcap_{n=0}^{\infty}\left(x_{\alpha}\right)^{n} R \text { if } \alpha \neq \bar{\alpha} \text { and } \alpha \text { is not a limit ordinal, } \\
& x_{\alpha+1} R=\bigcap_{\beta<\alpha} x_{\beta} R \text { if } \alpha \neq \bar{\alpha} \text { and } \alpha \text { is a limit ordinal. }
\end{aligned}
$$

That $\alpha_{0}=\bar{\alpha}$ is a consequence of the next lemma.

LEMMA 10. If $\bar{\alpha} \neq \alpha \in I$ or $\alpha=-1$ then $R \backslash R^{(\alpha)}=x_{\alpha+1} R$.

Proof. The proof is by transfinite induction. Clearly $R \backslash R^{(-1)}=x_{0} R$ since $x_{0} R$ is the unique maximal ideal of $R$. Let $\alpha \in I, \alpha \neq \bar{\alpha}$ and assume that $R \backslash R^{(\beta)}=x_{\beta+1} R$ if $\beta<\alpha$. If $\alpha$ is a limit ordinal then we obtain

$$
R \backslash R^{(\alpha)}=R \backslash\left(\bigcup_{\beta<\alpha} R^{(\beta)}\right)=\bigcap_{\beta<\alpha}\left(R \backslash R^{(\beta)}\right)=\bigcap_{\beta<\alpha} x_{\beta+1} R=x_{\alpha+1} R
$$

by $\left({ }^{*}\right)$ and the induction hypothesis. Now assume that $\alpha$ is not a limit ordinal. Then from $R \backslash R^{(\alpha-1)}=x_{\alpha} R$ it follows that $x_{\alpha}$ is a prime in $K_{\alpha-1}$. Also from (*) we obtain $x_{\alpha+1} K_{\alpha-1}=\bigcap_{n=0}^{\infty}\left(x_{\alpha}\right)^{n} K_{\alpha-1}$ and therefore $\operatorname{dim}_{k_{\alpha-1}}\left(x_{\alpha+1}\right)=\infty$. It follows that $x_{\alpha+1} \notin\left(K_{\alpha-1}\right)^{\prime}$. Therefore $x_{\alpha+1} \in R \mid R^{(\alpha)}$ and $x_{\alpha+1} R \subset R \backslash R^{(\alpha)}$. To show the reverse inclusion let $f \in R \backslash R^{(\alpha)}$. Then $f \in R \backslash R^{(\alpha-1)}=x_{\alpha} R$. In fact $f \in \bigcap_{n=0}^{\infty}\left(x_{\alpha}\right)^{n} R$. Otherwise $f=\left(x_{\alpha}\right)^{m} g$ where $m$ is the largest such integer. This implies $g \in R \mid x_{\alpha} R$ $=R^{(\alpha-1)}$ and so $g$ is a unit in $K_{\alpha-1}$. Since $x_{\alpha}$ is prime in $K_{\alpha-1}$ it follows that $\operatorname{dim}_{K_{\alpha-1}}$ $(f)<\infty$. Hence $f \in\left(K_{\alpha-1}\right)^{\prime} \cap R=R^{(\alpha)}$, a contradiction. Thus $f \in \bigcap_{n=0}^{\infty}\left(x_{\alpha}\right)^{n} R$, and so $f \in x_{\alpha+1} R$. Q.E.D. 
COROLlaRY. For each nonlimit ordinal $\alpha \leqq \alpha_{0}, x_{\alpha}$ is the unique (up to right unit factor) inf ${ }^{(\alpha)}$ prime in $R$.

Using the last corollary we may state Theorem 3 for the present case as follows.

THEOREM 4. Let $R$ be the ring of polynomials constructed in this section. Each nonzero element $f \in R$ can be written in the form $f=x_{\alpha_{1}}^{n_{1}} \cdots x_{\alpha_{k}}^{n_{k}} u$ where $n_{i}$ are positive integers, $\alpha_{1}>\cdots>\alpha_{k}$ and $u$ is a unit in $R$. This expression is unique in the sense that if $f=x_{\beta_{1}}^{m_{1}} \cdots x_{\beta_{h}}^{m_{h} v}$ is another such factorization of $f$, then $h=k, \alpha_{i}=\beta_{i}$ and $n_{i}=m_{i}$ $(i=1,2, \ldots, k)$.

Acknowledgement. The author is deeply indebted to Professor R. E. Johnson for encouragement and guidance in the development of the present work.

\section{BIBLIOGRAPHY}

1. N. Bourbaki, Éléments de mathématique. Algèbre commutative, Chapitre 2, Hermann, Paris, 1961.

2. P. M. Cohn, Noncommutative unique factorization domains, Trans. Amer. Math. Soc. 109 (1963), 313-331.

3. - Torsion modules over free ideal rings, J. London Math. Soc. 17 (1967), 557-599.

4. N. Jacobson, The theory of rings, Math. Surveys No. 2, Amer. Math. Soc., Providence, R. I., 1943.

5. A. V. Jategaonkar, A counter example in ring theory and homological algebra (to appear).

6. R. E. Johnson, Unique factorization in principal right ideal domains, Proc. Amer. Math. Soc. 16 (1965), 526-528.

7. O. Ore, Theory of noncommutative polynomials, Ann. of Math. (2) 34 (1933), 480-508.

UNIVERSITY OF NEW HAMPSHIRE,

DURhaM, New HampShiRe 